\title{
A generic procedure to monitor Maillard-derived fluorescent compounds in cookies by flow-injection analysis
}

\author{
Julia Calvarro · Vural Gökmen ·Francisco J. Morales \\ Consejo Superior de Investigaciones Científicas, ICTAN, Jose Antonio Novais 10, 28040 Madrid, Spain \\ Department of Food Engineering, Hacettepe University, Beytepe, 06800 Ankara, Turkey
}

\begin{abstract}
Flow-injection analysis is proposed for routine measurement of Maillard-derived fluorescent compounds (FC) in cookies as a marker of the extent of baking. In addition, procedure was applied to investigate the formation of free and total (bound to protein free) FC in cookie-resembling models and in commercial wheat-based cookies as well. FC accounts for the overall fluorescence response of Maillard-derived fluorescent compounds (bound or not to protein) formed during baking but not for a single compound. Free and total $\mathrm{FC}$ values increased exponentially during baking at 200,210 and $220^{\circ} \mathrm{C}$ and an induction period of 10 min was observed for free FC. In the complex scenario of the progress of the Maillard reaction (MR) during baking, formation of FC (347/4I5, ext/emm) was not the limiting step for browning development at the advanced stage of the reaction. Furthermore, the formation of Maillard- derived fluorescent compounds and browning during baking were a consequence of parallel reactions apart from the classical MR scheme of consecutive reactions. Total/ free FC ratio was dependent on the baking conditions applied and ratio was significantly decreased at severe baking conditions. Total/free FC ratio could be used as a reference marker for monitoring the process and to identify potential over-processing situations during baking. In addition, fluorescent residues were originally bound to protein because total/free FC ratio decreased drastically as increased the temperature and time of the process. Levels of total FC were nearly 20-fold of free FC in commercial samples. Values of FC were positively correlated with acrylamide, a Maillard-derived food processing contaminant.
\end{abstract}

KEYWORDS: Maillard reaction; Fluorescent compounds; Browning; Acrylamide; Cookie; Flow injection

\section{INTRODUCTION}

Unspecific procedures have been traditionally applied to monitor the advanced stage of the Maillard reaction (MR) during food processing and storage such as (I) by measuring of browning formation at $420 \mathrm{~nm}[\mathrm{I}, 2]$, and (2) using spectrophotometric tristimulus colour measurement [3, 4]. In the last decades, determination of Fluorescence has been proposed as an effective procedure to assess the extent of the MR as reviewed by Andersen and Mortensen [5] and Matiacevich et al. [6]. Besides to its application in food science, Fluorescence is also applied in biomedical area where MR takes places as well because Fluorescent compounds are related to the degree of the damage of biomolecules during an extended time frame. Then, Fluorescence profile is monitored in studies at physiological conditions in relation to glycosylation of proteins. The formation of the advanced glycosylation end products (AGEs) and subsequently AGE-related pathologies and chronic diseases, such as diabetes [7], lens ageing [8], atherosclerosis [9], including rheumatoid arthritis, haemodialysis-related amyloidosis [10], uraemia pathologies [I I], Alzheimer's [12], and other neurodegenerative diseases [13] are indirectly evaluated by Fluorescence measurement.

In contrast to the biomedical area where a substantial progress has been achieved, the chemistry of the formation of Fluorescent compounds derived from the MR in foods is still an infant and only few structures have been identified (for a review [14]). Morales et al. [15] and Morales and van Boekel [16] showed that a 
myriad of Fluorescent compounds derived from the MR could contribute to the overall Fluorescence in milk and milk-resembling systems after heating. Fluorescence compounds (FC) (Maillard-derived FC) were formed during heating of milk and milk-resembling systems. FC can be bound to the protein or not and bound to protein FC was quantitatively more important than free FC. Similar conclusion was also reached for breakfast cereal being the ratio total/free Maillard-derived Fluorescent compounds about tenfold and ratio total/free was regardless to the type of Fluor used in the formulation [17]. However, this situation might not to be necessarily equivalent in other food categories.

The route of formation of both FC (in foods) and Fluorescent AGE (in vivo) is not yet well understood. In some cases, Fluorescent compounds are concomitant in both scenarios such as pentosidine. As reviewed by Matiacevich et al. [6], Fluorescent compounds have been considered as intermediates or precursors during melanoidins formation and are valuable in helping to detect early stages of the reaction when there are no visible changes yet $[18,19]$. But other authors pointed out the possibility of Fluorescent compounds and melanoidins have independent kinetics, and colour and Fluorescence did not follow the same trend [20]. The Amadori rearrangement product undergoes dehydration and fission and yields colourless reductones as well as Fluorescent substances are formed and most of the structures remain unidentified. Several excitation and emission wavelengths have been proposed for characterizing and monitoring Maillard-derived Fluorescent products $(\mathrm{ext}=340-370 \mathrm{~nm}$ and $\mathrm{emm}=420-440 \mathrm{~nm}$, except for structures containing arginine, which present ext $=320 \mathrm{~nm}$ and $e m m=380 \mathrm{~nm}$ ) [6, 16, 21]. Morales and van Boekel [16] used the 347/415 ext/ emm for a generic measurement of FC, or 366/440 ext/ emm for pentodillysine, 335/385 ext/ emm for pentosidine has been used by Ferrer et al. [22].

Front-face Fluorescence spectroscopy (FFFS) can be directly applied to solid matrices in order to measure levels of MR-derived Fluorescent compounds [23] since FFFS measures Fluorescence emitted at the surface. Acquired signal can be calibrated for different food categories using an indirect correlation approach through chemometric tools, such as multivariate prediction models based on the multiway Parafac decomposition of 3D Fluorescence signal [5]. This approach has been successfully applied for investigating the effect of formulation and processing conditions in cookies [24]. However sophisticated equipments, sensors, software development, previous modelling design, and qualified personnel for data interpretation are needed.

Matiacevich et al. [6] identified that one of the limiting steps in the Weld of Maillard-derived Fluorescent compounds was the lack of a harmonised procedure for a routine measurement since there was not stable Fluorescent compounds identified yet for this purpose. In this context, Morales et al. [15] have suggested the possibility to apply measurement of free FC as a heat-induced index to assess nutritional and technological modifications during thermal treatment of foods. Lately, Birlouez-Aragon et al. [25] developed a smart procedure (FAST procedure) for distinguishing heat treatments and to estimate nutritional damage in milk products by connecting the FC values and losses of tryptophan as a marker of thermal denaturation of milk proteins.

In the present work, a simple, rapid and semi-automatised analytical procedure is proposed for routine measurement of Maillard-derived Fluorescent compounds in cookies. Flow-injection analysis (FIA) of both free and total FC was further applied in model cookies and commercial samples. In addition, routes of formation of both free and total (free + bound to protein) FC in cookies is discussed as well the relationship with acrylamide. 


\section{MATERIALS AND METHODS}

\section{Chemicals}

Sucrose, glucose, ammonium bicarbonate, pronase, sodium hydroxide, boric acid, acrylamide were purchased from Sigma (St. Louis, MO, USA). 13C3-Acrylamide (isotopic purity 99\%) was from Cambridge Isotope Laboratories (Andover, MA, USA). Ultra pure water was used throughout the entire experiments (MilliQ system, Millipore, Bedford, MA, USA). Methanol and acetonitrile (HPLC grade) were from Scharlau (Barcelona, Spain). Potassium hexacyanoferrate, zinc sulphate, formic acid (98\%) were of analytical grade and obtained from Panreac (Barcelona, Spain). Oasis HLB cartridges (30 mg, I mL) were supplied by Waters (Milford, MA, USA).

Dough preparation and baking conditions.

Wheat Fluor was supplied by local producers, and other ingredients were purchased from local supermarkets. Model cookie was prepared with $80 \mathrm{~g}$ of Fluor, I g of salt, $17.6 \mathrm{~g}$ of deionised water, $35.0 \mathrm{~g}$ sucrose, $0.8 \mathrm{~g}$ sodium bicarbonate, and $0.4 \mathrm{~g}$ ammonium bicarbonate. Dough $\mathrm{pH}$ value was 8.2 and average protein content was $4.53 \%(\mathrm{w} / \mathrm{w})$. The ingredients were thoroughly mixed and the dough was rolled out to disks with the diameter of $5.5 \mathrm{~cm}$ and the thickness of $2 \mathrm{~mm}$, and baked at $180,200,210$ and $220^{\circ} \mathrm{C}$ for 10 , 15, 20 and $25 \mathrm{~min}$ in a natural convection oven (Simsek Laborteknik, Turkey).

Commercial samples

Eleven samples of cookies from different producers were purchased in Spanish supermarkets. Only cookies with wheat-based Fluor in the formulation were used. Products containing chocolate, dried fruits or cream were excluded. A representative portion of cookies in each package were powdered in a grinder, homogenised and stored in polyethylene containers under vacuum at $4^{\circ} \mathrm{C}$ until analysed.

Extraction of free (FCF) and total (FCT) Fluorescence compounds

Samples were extracted according to Morales et al. [15] with some minor modifications. For analysis of FCF aqueous extraction was applied. BrieXy, $500 \mathrm{mg}$ of Wnely grinded sample were suspended in $5 \mathrm{~mL}$ of 20 $\mathrm{mM}$ phosphate buVer ( $\mathrm{pH} \mathrm{7.0)}$ in a $10 \mathrm{~mL}$ centrifugal tube. The tube was shaken vigorously for $2 \mathrm{~min}$ and clarified with $0.25 \mathrm{~mL}$ each of Carrez I (potassium ferrocyanide 15\% w/v) and Carrez II (zinc acetate 30\% $\mathrm{w} / \mathrm{v})$ solutions. The resulting mixture was centrifuged at $4,500 \mathrm{~g}$ for $10 \mathrm{~min}$ at $4{ }^{\circ} \mathrm{C}$. The supernatant was collected in a $10 \mathrm{~mL}$ volumetric Xask and two further extractions of the solid residue with $2 \mathrm{~mL}$ phosphate buVer were performed. The supernatants were pooled and the volume was made up to $10 \mathrm{~mL}$ with phosphate buffer. Solutions were diluted 10 -fold and Wltered before analysis.

For analysis of FCT (free + bound to protein) an enzymatic digestion and aqueous extraction was applied. A $100 \mathrm{mg}$ of sample was digested with $3 \mathrm{~mL}$ of a $0.375 \mathrm{mg} / \mathrm{mL}$ pronase $E$ solution $(1,500 \mathrm{U} / \mathrm{mL}$ in I M sodiumborate solution, $\mathrm{pH}$ 8.2) in a stoppered test tube for $36 \mathrm{~h}$ at $40{ }^{\circ} \mathrm{C}$ in a water bath under shaking. After cooling, the solution was centrifuged at $4,500 \mathrm{~g}$ for $10 \mathrm{~min}$ at $4{ }^{\circ} \mathrm{C}$. The supernatant was Witered $(0.45 \mathrm{~m})$ and made up with $10 \mathrm{~mL}$ of $20 \mathrm{mM}$ phosphate buffer $(\mathrm{pH}$ 7.0). A $50 \mathrm{~L}$ of solution was placed in an HPLC vial and $950 \mu \mathrm{L}$ of water was added.

Fluorimetric determination of FC by FIA

Samples were analysed within $18 \mathrm{~h}$ after extraction by FIA mode in an HPLC coupled to a spectrofluorimeter of high capacity of response. A Shimadzu HPLC system (Shimadzu Corporation, Kyoto, Japan) equipped with an LC-20AD pump, an LC-20AD/AT low-pressure gradient former, an SIL-IOADvp 
autosampler, a CTO-IOASVP oven, an SPD-M20A DAD detector, an RF-IOAxL Fluorescence detector controlled by a CBM-IOA communication bus module was used. LabSolutions software (vI.2ISP) was used. Samples $(40 \mu \mathrm{L})$ were injected in water at a flow of $0.5 \mathrm{~mL} / \mathrm{min}$ at $40{ }^{\circ} \mathrm{C}$. Detector was set at $347 \mathrm{~nm}$ of excitation and $415 \mathrm{~nm}$ of emission, $100 \mathrm{~ms}$ of response and gain $\times 4$ (sensitivity medium). Alternatively, absorption spectra and UV and visible range were simultaneously recorded between 190 and $800 \mathrm{~nm}$, and signal at $420 \mathrm{~nm}$ was recorded as well. The samples were run in triplicate and peak height was used for signal measurements. FC value is expressed as Fluorescence intensity (FI) per milligram of sample after appropriate correction for dilution. For internal reference of the $\mathrm{Fl}$ range and linearity, it was used a 0.05 $\mu \mathrm{g} / \mathrm{mL}$ solution of quinine sulphate in $0.01 \mathrm{~mol} / \mathrm{L}$ sulphuric acid. To evaluate precision, free and total fluorescent compounds in commercial samples were measured on three different days (in triplicate each day, day to day precision), in the same day (within-day precision, $n=5$ in triplicate), and for repeatability conditions $(n=8)$. The analyses are integrated within the scope of a certified laboratory controlled by AENOR (Spanish Association for Standardisation and Certification).

Spectrofluorimetric measurements

A fluorescence spectrophotometer (SMF-25, Kontron Instruments, Milan, Italy) was used for the determination of fluorescence. Fluorescence excitation spectrum and emission spectrum were recorded between 200 and $600 \mathrm{~nm}$. Quarzglass cuvettes (QS-I.000 Suprasil, Hellma GmbH \& Co, DE) with light path of $\mathrm{I} \mathrm{cm}$ were used.

LC-ESI-MS determination of acrylamide

Acrylamide was measured as described by Arribas-Lorenzo et al. [26]. The limit of quantitation was set at 20 $\mu \mathrm{g} / \mathrm{kg}$, precision (reproducibility) was lower than $12 \%$ and an average recovery of $93 \%$ was obtained.

Statistical analysis

Statistical significance of data $(P<0.05)$ were analysed by using Tukey's test (Statgraphics plus v5.I, 200I, Statistical Graphics Corp., Rockville, MD, USA). At least two independent analyses were carried out per sample.

\section{RESULTS AND DISCUSSION}

Different methods for measuring the formation of MR-derived Fluorescent compounds (FC) have been described in literature, which are lately adjusted according to the food category investigated and the extraction conditions applied [15, 2I]. Then, a pool of Fluorescent compounds was contributing in different extent to an overall unspecific fluorescence in the sample which was proportional to the modifications taking place during thermal treatment of the product. Unspecific fluorescence from FC, as done for AGEs in the biomedical Weld, has been mostly recorded at $347 \mathrm{~nm} / 415 \mathrm{~nm}(\mathrm{ext} / \mathrm{emm})$. Changes in the FI are correlated with changes in foods constituents during processing (food science Weld) or AGE-derived pathologies (biomedical Weld) $[25,27]$.

Excitation and emission spectra for a set of commercial cookies and model cookies were recorded in order to identify the most appropriate wavelength for analysis of FC in a cookie matrix. In all cases, a characteristic maximum about $420 \mathrm{~nm}$ for emission and two peaks around 340 and $279 \mathrm{~nm}$ for excitation were always observed as depicted in Fig. I. However, the ratio of response at 340 and $279 \mathrm{~nm}$ in the excitation scan was diVerent for FCF (Fig. Ia) and FCT (Fig. Ib) being the ratio lower after enzymatic digestion of the sample. In 
contrast, peak at $420 \mathrm{~nm}$ maintained its presence in both water soluble and enzymatically digested fraction. A partial contribution ( $<15 \%$ peak height) of the enzymes in the response at $280 \mathrm{~nm}$ in FCT was observed.

All studied samples showed the same profile, but variations in the ratio (height) between 340 and 279 peak was observed. These findings agree with our previous data for breakfast cereals [17] and milk [16], as well as the average values reviewed by Matiacevich et al. [6] who identified typical excitation wavelengths of 340$370 \mathrm{~nm}$ and emission wavelengths of $420-470 \mathrm{~nm}$ for most common Fluorescent structures. Thus, the excitation/emission wavelengths at $347 / 415 \mathrm{~nm}$ was maintained for cookies as a generic procedure for measuring Maillard-derived fluorescent compounds. In parallel, potential interferences from well-known fluorescence molecules in foods such as riboflavin (470/540, ext/ emm) or tryptophan (280/340, ext/ emm) or AGEs such as pentosidine (335/385, ext/emm) were also evaluated and were negligible at this conditions.

In addition, UV-VIS spectrum was recorded in the water soluble and enzymatically digested samples (data not shown). UV-VIS scan for FCF showed a maximum at $266 \mathrm{~nm}$ and signal for FCT shown a peak at 283 $\mathrm{nm}$ with a spectrum similar to furanic compounds [28].

Additional adjustments to the classical procedure in cuvette [15] were necessary before to settle the FIA. It is noteworthy to remain that higher dilution factor (at least 50 -fold) was applied to FCT samples as compared to FCF in order to avoid quenching phenomena and subsequently the underestimation of the emission FI with the presence of colored compounds. Data acquisition time was settled at 2 min and the peak was eluted at 0.56 min (Fig. 2). There were not significant differences between recording data as peak area or peak height (data do not shown), and peak height was chosen for practical reasons. Dilution factor and volume of injection were evaluated for FCF and FCT in order to ensure linearity in response. As previously stated by Morales and van Boekel [16], Maillard-derived Fluorescent compounds bound to protein are quantitatively more important that free ones, which makes the simultaneous measurement of browning and fluorescence a challenge since dilution applied for FCT in cookie samples was excessive for recording also browning at UV/VIS $420 \mathrm{~nm}$ in the same run. Sensitivity of Fluorescence detector is nearly 100 times higher than that of DAD. It was settled a sample injection volume of $40 \quad$ L (Fig. 3a) and an appropriate dilution factor of 0.05 (Fig. 3b) to ensure Fluorescence response within the linear range of measurement. Browning (UV/ VIS $420 \mathrm{~nm}$ ) and signal at $280 \mathrm{~nm}$ was recorded simultaneously to Fluorescence.

Procedure was in-house validated and evaluated for precision (repeatability and reproducibility) on three commercial cookies (SPI, SP2 and SP3) as summarised in Table I. Repeatability or (intra-assay precision) was evaluated in eight replicated at two levels of response. Reproducibility was evaluated at the same laboratory within different days and different personnel. Determination of FCT showed higher variability than FCF perhaps due to the need of additional treatment of the sample that imply additional sources of bias apart from the dilution factor applied. Owing to the nature of the analysis, recovery and specificity was not possible to be evaluated, since Fluorescent compounds counting for 347/475 ext/ emm cannot be put on a molar basis.

Procedure was applied in a cookie model system. FCF and FCT were analysed in a cookie model system baked under controlled conditions (Fig. 4). Baking was performed at different temperatures $(180,200,210$, $220^{\circ} \mathrm{C}$ ) for different times $(10,15,20,25 \mathrm{~min})$. Fluorescence increased exponentially with the baking conditions for FCF (Fig. 4a) and FCT (Fig. 4b) at 200, 210 and $220^{\circ} \mathrm{C}$ but, just slight changes were recorded at $180^{\circ} \mathrm{C}$. In contrast, a maximum of $\mathrm{Fl}$ after that a decay was not observed in model cookies at the baking 
conditions applied, as reported by some authors for sugar-amino acids/proteins model systems [18,19, 29]. Literature is controversial at this point because depending on the reaction conditions, such as $\mathrm{pH}$, temperature, water activity, and type of reactants, fluorescence might show a clear maximum after that a decay if heating is prolonged [30], or might increase continuously [3I], or to reach a plateau value [32]. The rate of formation of fluorescent compounds is governed by a number of variables that should be controlled [20]. These findings are useful for the application of FIA of Maillard-derived fluorescent compounds as a rapid procedure for monitoring extent of the MR in cereals products.

A short induction period was observed in the formation of FCF since not differences amongst temperatures were detected at 10 min of baking, but this response was not clear for FCT. It could be plausible that fluorescent compounds were previously formed at the protein backbone of flour protein and lately released according to the baking conditions. This hypothesis was also suggested by Morales and van Boekel [16] for milk-resembling systems, but it has not been proved yet. Morales et al. [15] also stated that free Maillardderived Fluorescent compounds in milk had two diVerent reaction routes depending on the presence of amino groups and that Fluorescence related to the MR is quantitatively most important. However, Fluorescent compounds, possibly with diVerent molecular structures, were also formed in trace amounts from sugar degradation. In cookies, both reactions are taking place simultaneously. It is noteworthy to mention that a residual Fluorescence response was recorded in the dough, 256 and 10,353 Fl/mg for FCF and FCT, respectively. However FCF and FCT were not detected or detected at traces in the Fluor (LOD 15 and $280 \mathrm{Fl} / \mathrm{mg}$ for FCF and FCT, respectively).

Browning as recorded at $420 \mathrm{~nm}$ (absorbance units $/ \mathrm{mg}$ ) was only measured in the aqueous extract of the FCF samples since samples enzymatically treated for FCT were more diluted and response of browning was not sensible enough at low temperatures of baking as explained formerly. As expected, browning increased with the baking conditions and an induction period up to $15 \mathrm{~min}$ was observed since coloured structures are formed at advanced stages of the MR. Bound to protein Maillard-derived Fluorescent compounds were always detected before chromophores were formed and they shown a dynamics with baking conditions indicating an apparent scenario of consecutive reactions are playing. Then, browning and Maillard-derived Fluorescence were positively correlated during baking of cookies as depicted in Fig. 5. Furthermore, the formation of Maillard-derived Fluorescent compounds was not the limiting step for occurrence of browning since FI did not decay with the time of baking. This fact is important and it could be plausible that parallel reactions, as opposite to the traditional scheme of consecutive reactions, may coexists; but with different reaction rates for the Fluorescent compounds and chromophores formation as suggested by Matiacevich et al. [6]. However, it was not observed a significant relationship between FCF and browning for commercial sample likely due to its heterogeneity in the formulation and baking conditions.

Flow-injection procedure for measurement of FCF and FCT was also applied on II commercial flour-dough cookies. Median values of 4,379 Fl/mg (I,320-13,683 min- max) and 100,243 Fl/kg (25,564-186,827 minmax) were obtained for FCF and FCT, respectively. Average ratio between FCT and FCF was nearly 20 -fold. Value of the ratio was double to the ratio reported in our previous findings for wheat-based breakfast cereals using cuvette analysis [17]. Ratio FCT/FCF was not linear and was significantly reduced from 20- to I0-fold as FCT value increased (Fig. 6). This observation could be due to several reasons: (I) there is a quenching effect for FCT, (2) net formation of FCT is reduced or net formation of FCF is increased, and (3) increase in the proportion of non-soluble material after enzymatic digestion. First option was discharged since the procedure was validated for linearity up to $320,000 \mathrm{Fl} / \mathrm{mg}$ for FCT. Next options are probable and 
cannot be excluded. It is known that at more severe baking conditions, the reaction rate of formation of high-molecular weight structures is enhanced and new polymers formed have less solubility.

In order to obtain more insight in this hypothesis, values for ratio FCT/FCF were correlated in the cookie model system as well as depicted in Fig. 7. Ratio FCT/FCF decreased significantly from 50-fold to nearly $10-$ fold at more severe baking conditions. The effect was time and temperature dependent for 200, 210 and 220 ${ }^{\circ} \mathrm{C}$. However, ratio FCT/FCF was maintained at $180{ }^{\circ} \mathrm{C}$ up to $25 \mathrm{~min}$. Ratio FCT/FCF could be used as reference for setting the limits to the appropriate range of processing and to avoid over-processing. These results are in agreement with the previous hypothesis that genesis of free Fluorescent compounds will be generated from bound to protein Fluorescent structures previously formed.

During baking, MR products with potential harmful effect are formed in carbohydrate rich foods such as acrylamide [33]. The main food commodities contributing to the acrylamide exposure were French fries, potato crisp, coffee, biscuit and bread while other food items contributed $<10 \%$ [34]. The presence of acrylamide as heat-induced toxicant led to public health concern and different National and International bodies aimed for monitoring, mitigation and for risk evaluation. Despite there is not regulation on this issue yet, it is important to develop rapid method for estimation of acrylamide formation in the production line since conventional analytical methods are expensive and time consuming since they are based in mass spectrometry. As an alternative, CIE L*a*b* colour system parameters or computer vision analysis have been used to confirm the correlation between the surface colour and the acrylamide concentration in different foods [35, 36]. Maillard-derived Fluorescent compounds in commercial cookies were compared with acrylamide content. Both free and total FC gave a significant correlation with acrylamide levels in cookies being $0.94 \mathrm{I}(\mathrm{P}<0.0 \mathrm{I})$ and $0.970(\mathrm{P}<0.0 \mathrm{I})$ for FCF and FCT, respectively (Fig. 8). Both indexes gave good correlation with acrylamide and for practical reasons, it was used the measurement of free Maillardderived Fluorescent compounds by FIA as an indirect marker of formation of acrylamide in cookies.

Under favourable reaction conditions (neutral $\mathrm{pH}$, salts, and reactants), Fluorescent products could be considered as adequate markers because they reflected the extent of the MR [20]. Thus, a rapid flowinjection procedure has been developed under controlled conditions for easily monitoring the progress of the MR. Procedure has been applied to gain knowledge on the nature of the free and bound to protein Maillard-derived Fluorescent compounds in cookies. A concomitant scenario with consecutive and parallel reactions in the formation of Maillard-derived Fluorescent substances and browning during baking of cookies cannot be excluded. Procedure reported for measurement of Maillard-derived Fluorescent compounds is easy to perform, rapid, accurate and require only very small quantities of sample and can be applied for monitoring the extent of the MR in cereals under controlled conditions.

Fluorescence associated with the extent of the MR in foods is not due to a unique Fluorescent substance and could be also polymeric structures because of that classical column chromatographic approaches are not suitable for this purpose as already shown by Morales and van Boekel [16]. The overall measure of Fluorescence response gave more precise information than browning on the changes produces during baking since detection is more sensitive to earlier stages of the reaction. FIA was adequate for a rapid monitoring of the extent of the baking in cookies including an estimation of acrylamide formed. In future, rapid measurement of Maillard-derived Fluorescent compounds could be a useful tool to estimate the impact of several sources of variation in the formulation, such as type of leavening agent, Fluors, Wbre and baking conditions on formation of certain heat-induced contaminants. 


\section{ACKNOWLEDGMENT}

The authors are indebted to D. Gómez, for her technical assistance. This research was partly supported by the Spanish Ministry of Education ad Science (project AGL2005-01735) and Turkish Academy of Science (GEBIP Study Grant), Hacettepe University Scientific Research Unit (Project no 00IG007). Ms. Calvarro was recipient of a grant from the University San Pablo CEU (Madrid).

\section{REFERENCES}

I. Friedman M (1996) J Agric Food Chem 44:63I-653

2. Palombo R, Gertler A, Saugy I (1984) J Food Sci 49:1609-1613

3. Rampilli M, Andreini R (1992) Ital J Food Sci 4:285-29I

4. Pagliarini E, Vernile M, Peri C (1990) J Food Sci 55:1766-1767

5. Andersen CM, Mortensen G (2008) J Agric Food Chem 56:720-729

6. Matiacevich SB, Santagapita PR, Buera MP (2005) Critical Reviews Food Sci Nutr 45:483-495

7. Vlassara H, Brownlee M, Cerami A (1986) Clinical Chem 32:B37-B4 I

8. Monnier VM, Cerami A (198I) Science 211:491-493

9. Song DU, Jung YD, Chay DO, Lee KH, Yang SY, Shin BA, Ahn BW (2002) Arch Biochem Biophys 397:424-429

10. McDonald SP, Coates PTH, Disney APS (1998) Ann Rheum Dis 57:193-195

II. Raj DS, Choudhury D, Welbourne TC, Levi M (2000) Am J Kidney Dis 35:365-380

12. Smith MA, Taneda S, Richey PL, Miyata S, Yan SD, Stern D, Sayre L, Monnier VM, Perry G (1994) Proc. Natl Acad Sci USA 91:57I0-57/4

I3. Sell DR, Monnier VM (1989) J Biol Chem 264:21597-21602

14. Henle T (2005) Amino Acids 29:3 13-322

15. Morales FJ, Romero C, Jiménez-Pérez S (1996) Food Chem 57:423-428

16. Morales FJ, van Boekel MAJS (1997) Int Dairy J 7:675-683

17. Delgado-Andrade C, Rufián-Henares JA, Morales FJ (2006) Mol Nutr Food Res 50:799-804

18. Namiki M, Oka M, Otsuka M, Miyazawa T, Fujimoto K, Namiki K (1993) J Agric Food Chem 41:17041709

19. Labuza TP (1994) In: Maillard reactions in chemistry, Food and Health. Pp. I76-18I. The Royal Society of Chemistry, Cambridge.

20. Matiacevich SB, Buera MP (2006) Food Chem 95:423-430

21. Ferrer E, Alegría A, Farré R (2000) Rec Res Develop Agric Food Chem 4:269-290

22. Ferrer E, Alegría A, Farré R, Clemente G, Calvo C (2005) J Agric Food Chem 53:49II-4917

23. Birlouez-Aragon I, Locquet N, Louvent ED, Bouveresse DJR, Stahl P (2005) Ann New York Acad Sci 1043:308-318

24. Rizkallah J, Morales FJ, Ait-Ameur L, Fogliano V, Hervieu A, Courel M, Birlouez-Aragon I (2008) Chemometrics Intell Lab Syst 93:99-107

25. Birlouez-Aragon I, Leclère J, Quedraogo CL, Birlouez E, Grongnet JF (200I) Nahrung/Food 45:20I-205

26. Arribas-Lorenzo G, Fogliano V, Morales FJ (2009) Eur Food Res Technol 229:63-72

27. Meerwaldt R, Lutgers HL, Links TP, GraaV R, Baynes JW, Gans ROB, Smit AJ (2007) Diabet Care 30:107-112 
28. Rufián-Henares JA, Delgado-Andrade C, Morales FJ (2006) J AOAC Int 89:161-195

29. Benjakul S, Lertittikul W, Bauer F (2005) Food Chem 93:189- 196

30. Baisier WM, Labuza TP (1992) J Agric Food Chem 40:707-713

31. Cerruti P, Resnik SL, Seldes A, Ferro-Fontán C (1985) J Food Sci 50:627-636

32. Kato Y, Matsuda T, Kato N, Nakamura R (1989) J Agric Food Chem 37:1077-108I

33. Tareke E, Rydberg P, Karlsson P, Eriksson S, Tornqvist M (2002) J Agric Food Chem 50:4998-5006

34. Boon PE, Mul A, van der Voet H, van Donkersgoed G, Brette M, van Klaveren JD (2005) Mutat Res Genet Toxicol Environ Mutagenesis 580:143-155

35. Pedreschi F, Moyano P, Kaack K, Granby K (2005) Food Res Int 38: I-9

36. Gökmen V, Acar OC, Arribas-Lorenzo G, Morales FJ (2008) J Food Eng 87:380-385 
FIGURES AND TABLES

Table I. Repeatability and reproducibility for the determination of free and total Maillard-derived fluorescent compounds (FC) in cookies by flow-injection analysis

\begin{tabular}{|c|c|c|c|c|c|c|}
\hline & \multicolumn{3}{|c|}{$\mathrm{FC}_{\mathrm{F}}(\mathrm{FI} / \mathrm{mg})$} & \multicolumn{3}{|c|}{$\mathrm{FC}_{\mathrm{T}}(\mathrm{FI} / \mathrm{mg})$} \\
\hline & Mean & $\mathrm{SD}$ & RSD & Mean & SD & RSD \\
\hline \multicolumn{7}{|c|}{ Repeatability } \\
\hline SP1 & 1,208 & 23 & 1.9 & 28,056 & 358 & 2.0 \\
\hline SP2 & 1,295 & 12 & 0.9 & 24,270 & 313 & 1.5 \\
\hline SP3 & 4,348 & 27 & 0.6 & 72,826 & 1,714 & 2.4 \\
\hline \multicolumn{7}{|c|}{ Within day } \\
\hline SP3 & 4,379 & 114 & 2.6 & 74,966 & 2,104 & 2.8 \\
\hline \multicolumn{7}{|c|}{ Day to day } \\
\hline SP3 & 4,424 & 182 & 4.1 & 73,076 & 5,224 & 7.1 \\
\hline
\end{tabular}

$F I$ fluorescence intensity, $R S D$ residual standard deviation, independent commercial samples (SP1, SP2 and SP3) 
Figure I. Fluorescence spectra of FCF (a) and FCT (b) from a model cookie baked at $210^{\circ} \mathrm{C}$ for $20 \mathrm{~min}$. Excitation spectra ( emm $=415 \mathrm{~nm})$, emission spectra $(\mathrm{ext}=347 \mathrm{~nm})$
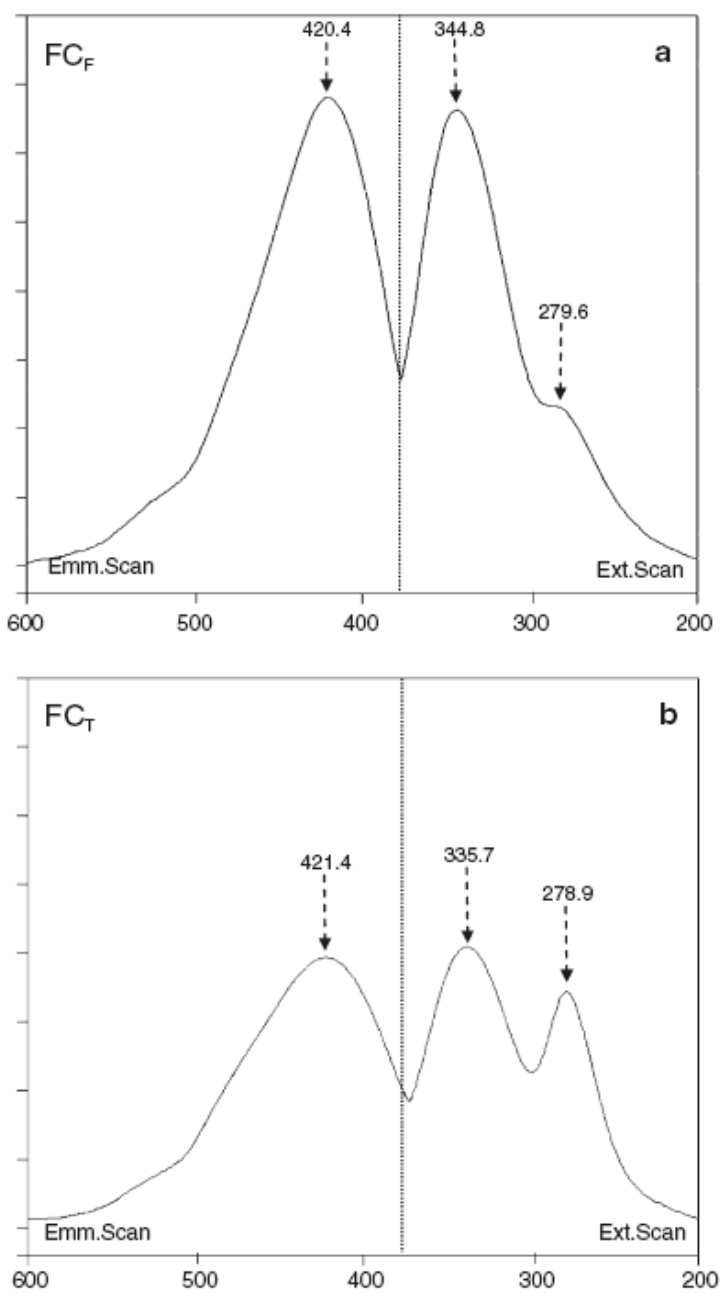
Figure 2. Measurement profile of four commercial cookies by the flow injection fluorimetric method

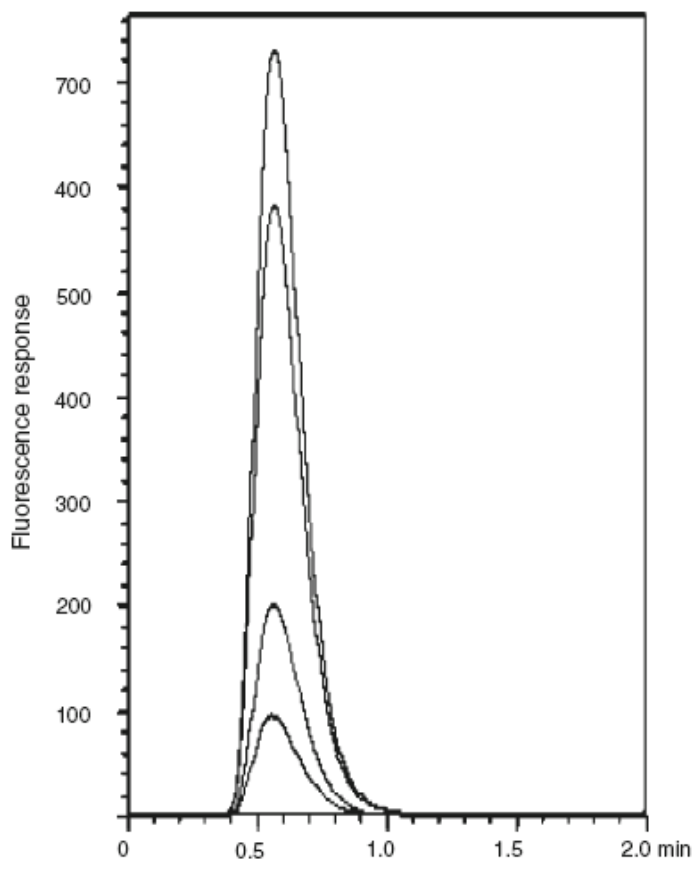


Figure 3. Effect of volume of injection (a) and dilution factor applied (b) on the peak height for fluorescence, $420 \mathrm{~nm}$ and $280 \mathrm{~nm}$. Browning (absorbance at $420 \mathrm{~nm}$ ) displayed at the secondary $Y$ axes
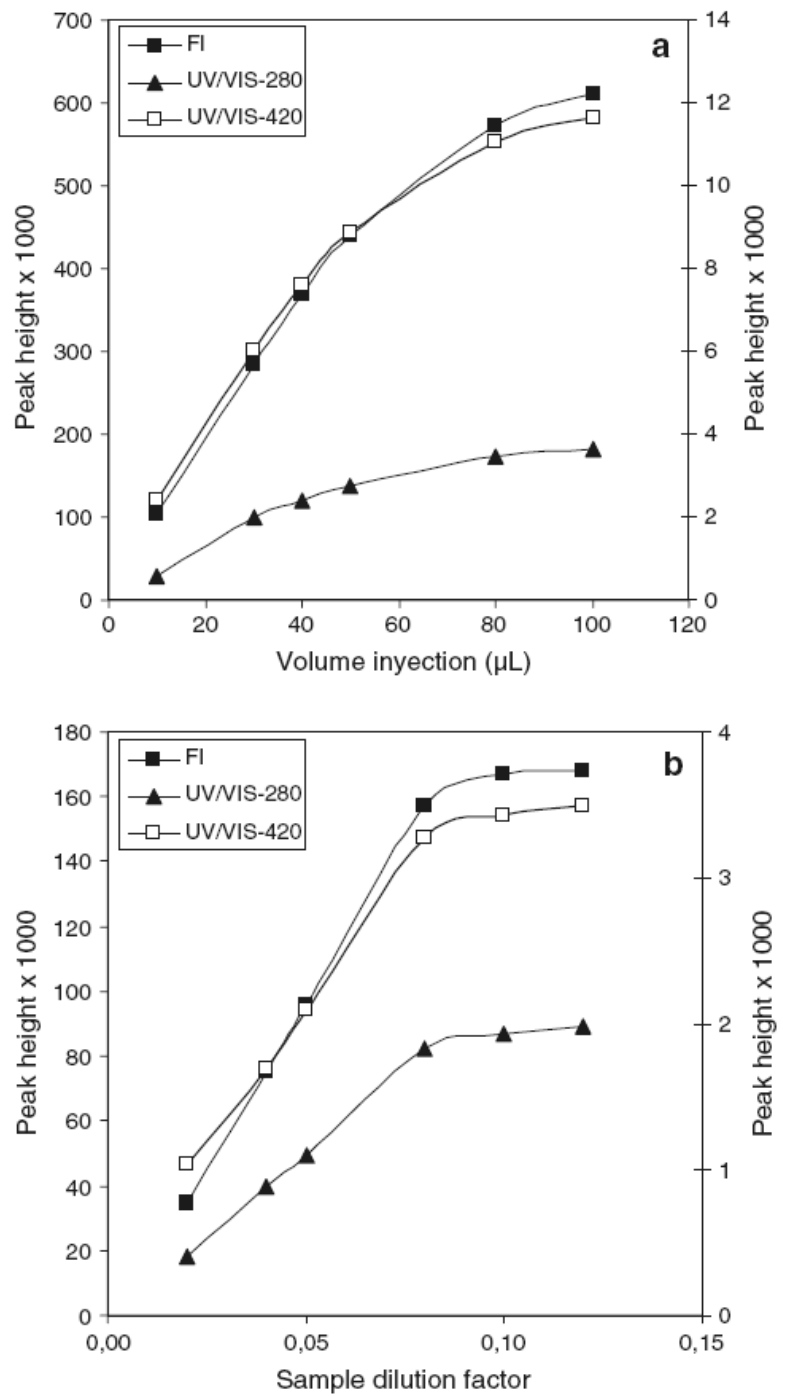
Figure 4. Effect of baking on the levels of FICF (a) and FICT (b). Error bar from two independent sample preparation
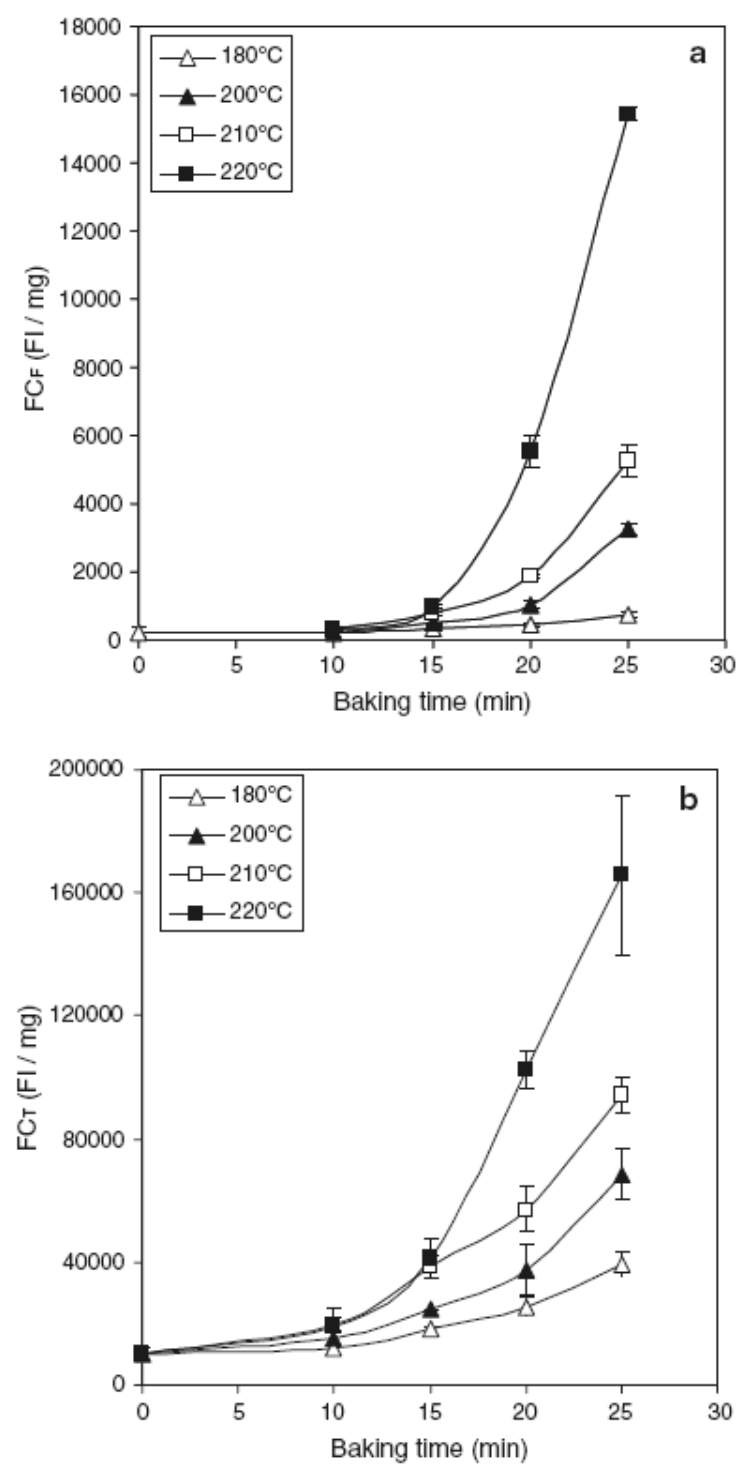
Figure 5. Relationship between browning (absorbance at $420 \mathrm{~nm}$ ) and free Maillard-derived fluorescent compounds (FCF) in model cookies baked under controlled conditions

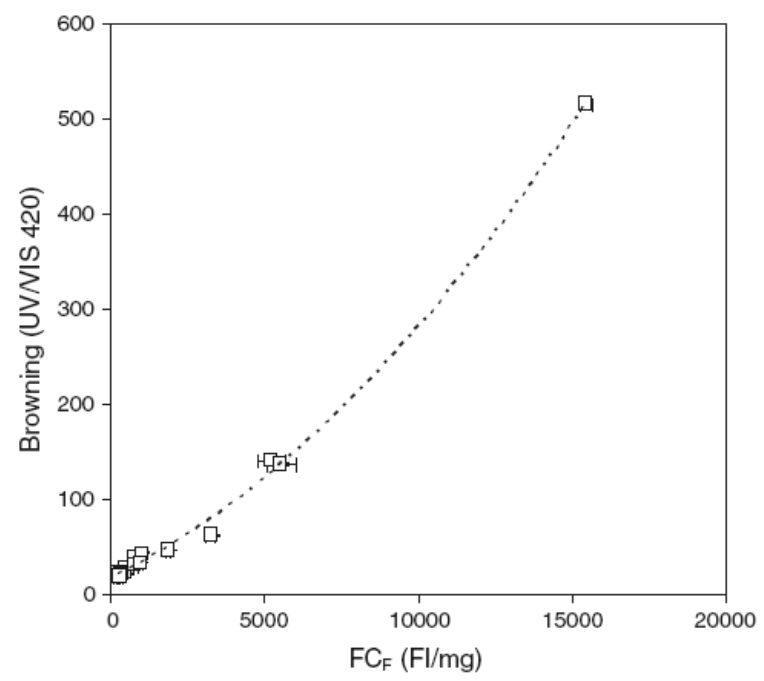


Figure 6. Relationship between FCT and FCF in commercial cookies

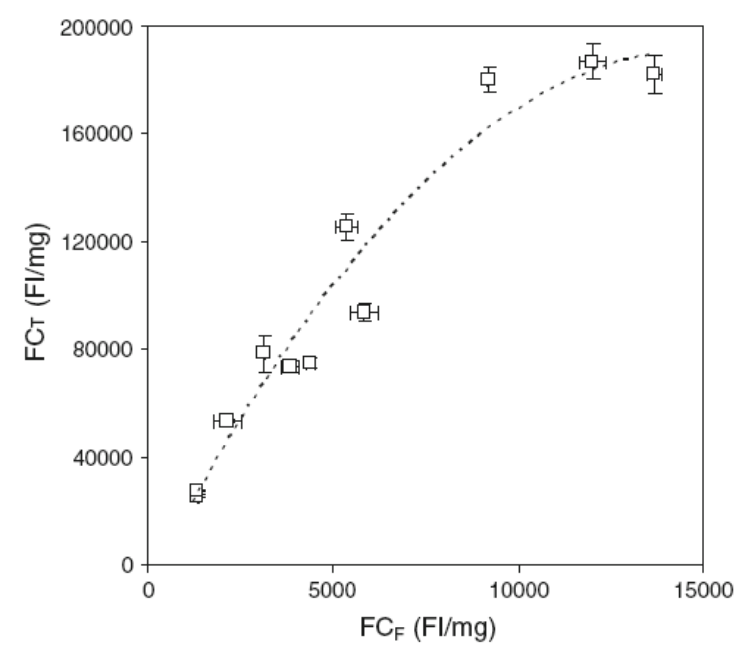


Figure 7. Dynamics of the ratio FCT/FCF with the baking time at $180,200,210$, and $220^{\circ} \mathrm{C}$ in model cookies

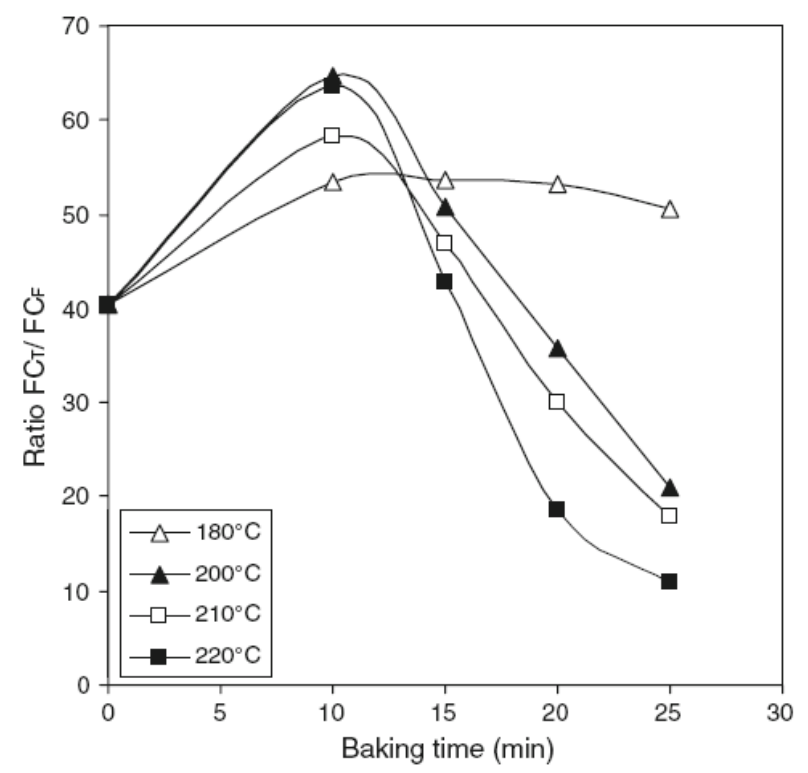


Figure 8. Relationship between FCF (a) and FCT (b) and acrylamide in commercial cookies
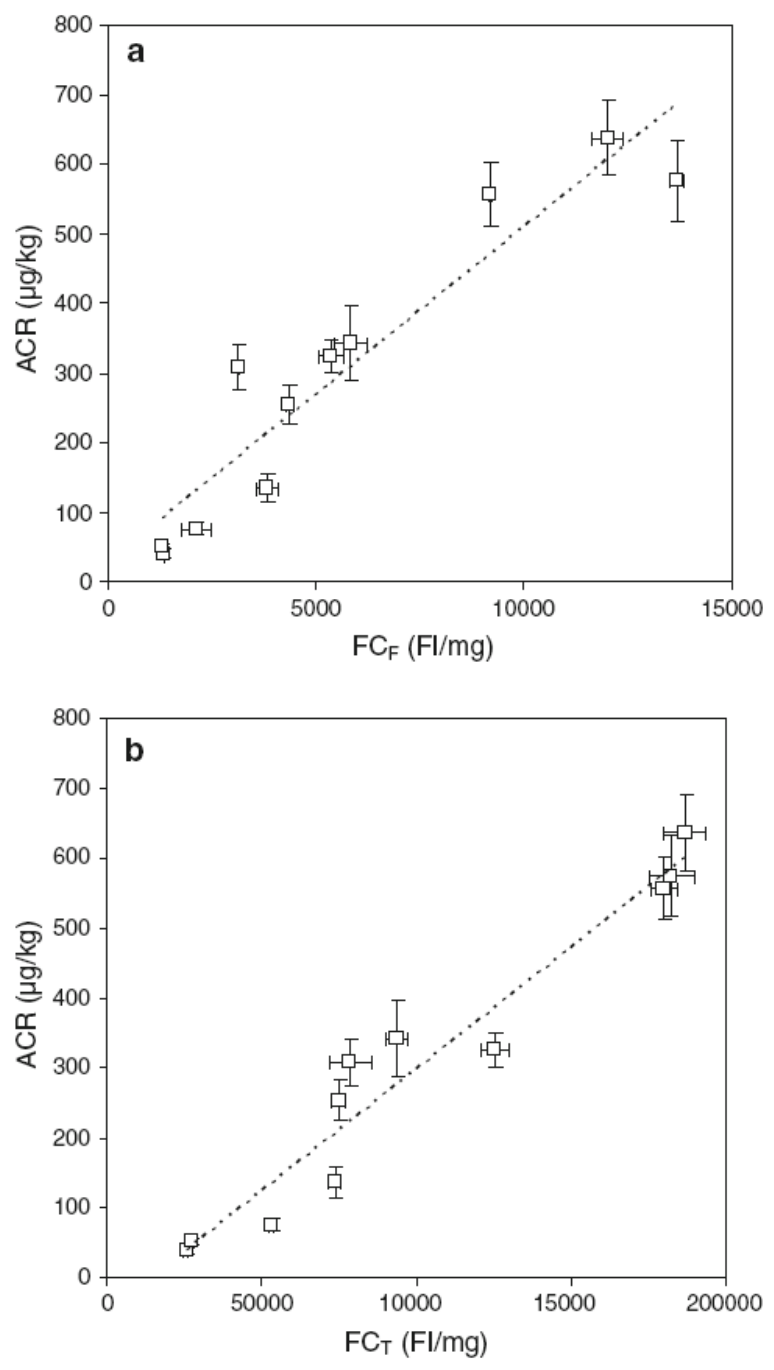